\title{
Formação para Atenção Básica: Relato de uma Experiência Docente
}

Medical training for Primary Health Care: a Teaching Experience Report

Formación para Atención Primaria em Salud: Relato de una Experiencia Docente

Marcia Cavalcanti Raposo Lopes ${ }^{1}$

\section{Resumo}

Objetivo: apresentar uma experiência docente vivida na primeira turma do Mestrado Profissional em Saúde da Família, problematizando questões que nos permitam avançar no seu desenvolvimento. Tal mestrado se constitui em estratégia importante para fortalecer a formação médica voltada para o SUS e, mais especificamente, para a Atenção Básica (AB). Ele institui um formato inovador no campo da saúde coletiva tanto por ser um Mestrado em rede que permite sua entrada simultânea em todas as regiões do país, quanto por seu caráter semipresencial com grande parte de sua carga horária em EAD. Método: Relata- se o processo de ensino-aprendizagem da disciplina de promoção de saúde a partir do desenvolvimento de tarefas que envolvem o trabalho dos profissionais no território. Resultados: Destaca-se o potencial do formato do curso e sua capacidade de interlocução com a prática dos mestrandos, favorecendo que o mestrado cumpra sua função no fortalecimento da formação para $\mathrm{AB}$ no Brasil. Conclusão: Sistematizar uma avaliação das potencialidades e dos entraves deste processo tão inovador.

Descritores: Educação em Saúde; Atenção Primária à Saúde; Promoção da Saúde.

${ }^{1}$ Psicóloga, Mestre em Saúde Coletiva pelo IMS/UERJ e Doutora em Psicologia Social pela UERJ. Professora-pesquisadora da EPSJV/Fiocruz. Autora correspondente: Rua Alice, 194/803. Laranjeiras, Rio de Janeiro - RJ, Brasil.22241-020. Email: mlopes@fiocruz.br

Recebido: Abr. /2018 - Aceito: Ago. /2018. 


\section{Abstract}

This report presents a teaching experience with the first group of the Professional Master in Family Health Programme. This master degree constitutes an important strategy to strengthen the medical training for Primary Health Care in brasilien public health sistem (SUS). This course has innovative features. First, it is a distance learning Master. Second, it is developed by a network of universities. It allows simultaneous students admission in all regions of our country. The text presents health promotion class. In it, students develop tasks related to their work in the territory. The experience shows that this kind of course can potentiate the medical training for Primary Health Care in Brazil. Conclusions: Systematize an assessment of the potentialities and obstacles of this innovative process.

Descriptors: Health Education; Primary Health Care; Health Promotion.

\section{Resumen}

Este trabajo objetiva presentar una experiencia docente vivida en la primera clase del Máster Profesional en
Salud de la Familia, problematizando cuestiones que nos permitan avanzar en su desarrollo. Tal curso se constituye en una estrategia importante para fortalecer la formación médica orientada al SUS y, más específicamente, para la Atención Primaria en Salud (APS). El instituye un formato innovador en el campo de la salud colectiva, por ser una Maestría en red que permite su entrada simultánea en todas las regiones del país y por su carácter semipresencial con gran parte de su carga horaria en EaD. Se relata el proceso de enseñanza-aprendizaje de la disciplina de promoción de la salud a partir del desarrollo de tareas que involucran el trabajo de los profesionales en el territorio. Se destaca el potencial del formato del curso y su capacidad de interlocución con la práctica de los maestrandos lo que favorece que el curso cumpla su función en el fortalecimiento de la formación para APS en Brasil. Conclusiones: Sistematizar una evaluación de las potencialidades y los obstáculos de este proceso tan inovador.

Descriptores: Educación en Salud; Atención Primaria de Salud; Promoción de la Salud. 


\section{Introdução}

Construído a partir da herança de um sistema embasado em práticas curativas e hospitalocêntricas, o SUS enfrenta inúmeros desafios para a implementação da Atenção Primária em Saúde (APS) abrangente ${ }^{(1)}$. O desfinanciamento público, as disputas em torno da mercantilização da saúde e as dificuldades próprias de estruturação de um sistema nacional de saúde em um país heterogêneo com dimensões continentais se constituem em entraves gigantescos para a consolidação de um sistema universal de saúde e de um modelo de atenção que adote uma lógica de atenção longitudinal centrada na pessoa, na família, na comunidade e no território. No entanto, para isto é necessário ressaltar questões como a organização dos serviços, a oferta e a formação dos trabalhadores de saúde, em especial, dos médicos, como pontos fundamentais para $\mathrm{o}$ fortalecimento deste modelo.

Muitos têm sido os debates sobre estas questões que nos aparecem de forma bastante entrelaçada, já que a transformação da organização e da lógica dos serviços não acontece por decreto, mas envolve o trabalho cotidiano dos profissionais de saúde. Neste sentido, é claro que as condições de trabalho, a presença contínua dos profissionais e suas noções de saúde, doença e cuidado - logo, sua formação são aspectos centrais desta transformação.

Gomes et $\mathrm{al}^{(2)}$. analisaram a questão da formação médica em relação à Atenção Primária em Saúde e apontaram as dificuldades do modelo hegemônico de formação médica concentrado principalmente no âmbito hospitalar com ênfase nos processos biológicos do adoecimento onde se constrói o conhecimento da doença e não do sujeito e de seu ambiente familiar e social.

Jesus, Medina e $\operatorname{Prado}^{(3)}$ destacaram como ao longo da trajetória da implementação da APS (chamada em nosso país de Atenção Básica $(\mathrm{AB})$ no Brasil, apesar das iniciativas para atração e fixação de profissionais de saúde, poucos resultados foram obtidos em relação a superação da iniquidade distributiva dos trabalhadores e sua fixação em regiões de maior vulnerabilidade social. Em relação especificamente à formação, Campos ${ }^{(4)}$ também apontou as limitações das políticas propostas nas quais muitos dos esforços têm dificuldades em avançar ou recaem sobre o ombro 'autônomo' e frágil dos municípios. 
É neste cenário de busca de soluções para a consolidação da atenção básica, impulsionado pelo movimento de junho de 2013 e suas reivindicações em relação à saúde pública, que se instituiu o Programa Mais Médicos (PMM). Com este Programa, entre outros pontos, o governo Federal de então, assume um lugar de protagonismo na reorientação da formação médica e na definição da lógica de expansão de vagas de graduação em Medicina que passa a ser regulada e planejada pelo Estado em função da necessidade social da oferta da formação médica.

Salientam-se, aqui, dois importantes efeitos das medidas propostas por este Programa: a criação de novas escolas médicas com destaque para aquelas que se instituíram em regiões distantes dos grandes centros urbanos e a ampliação da Atenção Básica como cenário de ensino aprendizagem seja durante a graduação, seja no momento da residência médica.

Para que estes processos se consolidem, entretanto, é necessário mais do que somente a reorganização formal do Ensino Médico. Como nos lembram Gomes et al. ${ }^{(2)}$ ao discutir a formação médica na Atenção Básica, para que o estudante possa se desenvolver e aprender a realizar um cuidado de forma integral, é preciso que não só a gestão local, mas também profissionais da Estratégia Saúde da Família (ESF) (especialmente se atuam como preceptores) e docentes compreendam seu papel no processo pedagógico inerente a inserção do estudante nos serviços de saúde.

Assim, um importante investimento para a melhor estruturação destas experiências é promover a formação de docentes e preceptores comprometidos com a construção do SUS e da Atenção Básica que queremos - profissionais ainda escassos no campo da saúde, especialmente se considerarmos as localidades mais distantes que mais necessitam da formação de médicos. Para atender esta demanda, se estruturou a proposta do Mestrado Profissional em Saúde da Família (Profsaude) - curso em rede nacional, desenvolvido por 20 (vinte) instituições de ensino e pesquisa de todas as regiões do país. Financiado pelos Ministérios da Saúde e da Educação, sua primeira turma envolve cerca de 150 professores e 200 alunos. Uma proposta complexa e diferenciada que compõe o pano de fundo da experiência aqui relatada.

Considerando que a Política Nacional de Atenção Básica publicada em $2017^{(5)}$, tende a produzir um 
importante retrocesso no desenho da APS no Brasil ${ }^{(6)}$, este Mestrado torna-se uma estratégia ainda mais importante, constituindo-se em espaço de formação crítica de formadores, portanto, ferramenta para o fortalecimento da luta por uma Atenção Primária em Saúde consistente com os princípios do SUS.

Dessa forma, objetivo deste trabalho foi apresentar uma experiência de formação vivida na primeira turma deste curso, problematizando questões que nos permitam avançar no seu desenvolvimento. Assim, pretendemos relatar o processo de trabalho que se constituiu a partir da disciplina de Promoção da Saúde, as propostas metodológicas, o desenvolvimento do tema e o processo de ensinoaprendizagem disparado pelo curso, tomando como ponto especial o fortalecimento de uma Atenção Básica de qualidade no SUS. Importante ressaltar que seu formato em rede e sua metodologia em EAD - características absolutamente inovadoras no campo da saúde coletiva - propiciaram uma experiência importante de garantir a oferta de formação em todo o território nacional e com um grande número de mestrandos por turma.

\section{Método}

A proposta de Mestrado Profissional (MP) no Brasil surge em 1995. Ela está relacionada com a construção de um esforço para aproximar a pesquisa do exercício profissional e tem como desafio integrar a investigação científica ao ambiente profissional dos estudantes, favorecendo a reflexão e a intervenção sobre a sua realidade laboral.

Seguindo esta proposta, o Profsaúde se coloca como objetivos ${ }^{(7)}$ :

Formar profissionais de saúde para exercerem atividades de docência, preceptoria e gestão; formar profissionais de saúde para exercerem atividades de investigação e de ensino nas unidades de saúde; fortalecer as atividades de produção do conhecimento e ensino na Saúde da Família nas diversas regionais do país; qualificar o profissional do Programa Mais Médicos para o trabalho no Saúde da Família/AB; articular elementos da educação, atenção, gestão e investigação no aprimoramento da ESF e estabelecer uma relação integradora entre 0 serviço de saúde, os trabalhadores e os usuários.

O curso é semi-presencial e, portanto, desenvolvido primordialmente em Educação a Distância (EaD). Seu currículo prevê nove disciplinas obrigatórias, sendo, oito desenvolvidas módulos comuns a todas as instituições parceiras e uma, construída de forma singular por cada uma delas, em função da especificidade de sua turma e de sua região do país. O aluno precisa, ainda, 
cumprir dez créditos eletivos e 22 créditos referentes ao desenvolvimento da dissertação. Além dos trabalhos online estão previstos durante o curso 8 encontros físico-presenciais (seis no primeiro ano, enquanto os alunos estão realizando as atividades em $\mathrm{EaD}$ e dois, no segundo ano)

A proposta das disciplinas comuns é construída em função de três eixos fundamentais - Atenção, Educação e Gestão, além da elaboração do trabalho final do curso. Assim, o primeiro eixo foi constituído pelas disciplinas "Atenção Integral na Saúde da Família" e "Atenção e Gestão do Cuidado"; o segundo eixo, pelas disciplinas de "Educação na Saúde" e "Promoção da Saúde" e abordando o terceiro eixo, as disciplinas "Planejamento e Avaliação na Saúde da Família" e "Sistema de Informação no Cuidado e na Gestão”. Para possibilitar o acompanhamento da elaboração do projeto e da dissertação são oferecidas outras duas disciplinas metodológicas Produção do Conhecimento em Serviços de Saúde e Seminários de Acompanhamento.

As atividades $\mathrm{EaD}$ são desenvolvidas no Ambiente Virtual de Aprendizagem (AVA) - Open Source Moodle (Modular Object Oriented Distance Learning - Objeto Modular
Orientado ao Ensino a Distância) que possibilita ações educativas compartilhadas através da utilização de tecnologia. Todo o conteúdo do curso foi construído buscando relacionar a produção do conhecimento com a prática nos serviços de saúde ou nas universidades e tomando o estudante como o sujeito de sua própria aprendizagem.

\section{A Disciplina de Promoção da Saúde e suas Propostas Metodológicas}

A disciplina de Promoção da Saúde, como dito anteriormente, faz parte do eixo educação. Ela se inicia no segundo semestre do curso e pressupõe que os alunos desenvolveram durante o primeiro semestre uma discussão teórica e metodológica sobre educação, tendo se aproximado da obra de Paulo Freire e da concepção de Educação Popular.

É a partir daí que se toma como proposta já na primeira semana de aula $^{(8)}$ :

Discutir os princípios da Promoção da Saúde no contexto dos territórios e compreender a possibilidade de processos de produção da saúde em condições adversas, considerando os sentidos e significados que as pessoas atribuem à saúde, à vida e à promoção da saúde. 
Para tanto, o curso faz inicialmente a discussão de textos teóricos sobre Promoção da Saúde, problematizando uma atenção de base puramente biomédica e ressaltando a importância do conhecimento do território onde se atua e a escuta dos usuários. As ferramentas utilizadas no AVA são Fórum e Wiki, que permitem, de maneira diferente, a troca virtual entre os alunos e o docente.

Após a discussão teórica, a disciplina propõe que os alunos identifiquem nos Planos Municipais de Saúde de seus municípios e nas atividades desenvolvidas em suas unidades de saúde, as ações de promoção da saúde.

Além disso, os alunos são estimulados a perceberem a atuação de agentes informais de saúde e a existência de pontos de cuidado e acolhimento fora do sistema, que precisam ser valorizados. A disciplina propõe entre suas atividades, uma entrevista com um destes agentes.

Numa terceira etapa, a disciplina traz para o centro do debate o usuário e suas dificuldades para o autocuidado, sendo uma das tarefas propostas entrevistar usuários que convivam com doenças crônicas sobre seu processo de adoecimento e a forma que construiu para seguir a vida convivendo com os limites trazidos por este.

Para finalizar as discussões trazidas pela disciplina se discute criticamente a educação em saúde "prescritiva" e se propõe que os alunos construam conjuntamente uma proposta de trabalho pedagógico com estudantes de graduação/residentes voltada para sua atuação com pacientes crônicos.

Durante todo o semestre a troca virtual com os alunos foi é feita principalmente através de Fóruns.

\section{Resultados e Discussão}

A noção de Promoção da saúde é ponto fundamental da proposta da Atenção Primária à Saúde abrangente. Ela traz consigo importantes debates que envolvem analisar criticamente a racionalidade biomédica e suas limitações no que tange a atenção sanitária, inserindo no campo da saúde temas como determinantes sociais da saúde, educação em saúde, práticas informais de saúde e participação social.

Muitas vezes, estes são temas pouco valorizados pelos médicos no seu cotidiano de trabalho, já que eles ficam premidos pelo excesso de demanda por atendimentos clínicos tradicionais. Neste sentido, a disciplina se constituiu numa importante oportunidade para 
modificar a prática médica nas unidades de saúde.

A discussão teórica sobre promoção da saúde, feita no início do curso, permitiu uma primeira aproximação do conceito ainda muito colado à ideia de prevenção de doenças. As estratégias online utilizadas e a pouca prática de alunos e docente com elas não favorecerem, entretanto, uma discussão mais aprofundada sobre o tema.

$\mathrm{Na}$ sequência do curso, com as diferentes tarefas desenvolvidas pelos estudantes a partir de sua realidade de trabalho, contudo, a disciplina foi ganhando consistência. A possibilidade que os mestrandos tiveram de levantar e apresentar as atividades de promoção da saúde desenvolvidas em suas unidades de saúde, conhecer melhor as práticas de cuidado informal do território, escutar os usuários com doenças crônicas através de entrevistas sobre seu processo de adoecimento, entre outras atividades se constituiu num importante espaço de análise das práticas realizadas na $\mathrm{AB}$ e de desenvolvimento dos estudantes.

É importante ressaltar que muitos estudantes investiram bastante na execução das suas tarefas e construíram interessantes análises sobre os efeitos de seu trabalho, mas a realização de uma troca mais vigorosa entre eles foi dificultada pela maneira como processo virtual se construiu, só acontecendo mais efetivamente no momento presencial.

Ponto alto do curso, estes momentos construídos a partir dos processos disparados pelo período online, se constituíram em espaço para discussões críticas e produção de conhecimento. O debate teórico fica enriquecido pela diversidade de experiências dos alunos, favorecendo a possibilidade de problematizar criticamente a maneira como, muitas vezes, os trabalhos de promoção da saúde acabam se constituindo em tentativas de impor "estilos de vida saudáveis" aos pacientes. Não há como não valorizar o momento do encontro físico entre mestrandos e professores, fazendo nos salientar a importância deles para os processos pedagógicos.

Evidentemente, todo este processo não transforma as práticas nas unidades de saúde, nem garante que os profissionais formados não exercerão seu trabalho sem serem atravessados pelas diferentes contradições discutidas no curso sobre as ações de promoção de saúde e mais amplamente da $\mathrm{AB}$ no Brasil. É muito difícil escapar da lógica puramente biomédica e da proposição vertical de hábitos 
saudáveis tão arraigadas no nosso sistema de atenção à saúde. No entanto, a aproximação dos agentes informais de saúde e a escuta dos usuários e suas dificuldades em realizar os tratamentos propostos não se dão sem deixar marcas. $E$ isto foi abertamente relatado por alguns dos estudantes. Neste ponto é preciso destacar o potencial do formato do curso e sua capacidade de interlocução com a prática dos mestrandos, favorecendo que o mestrado cumpra sua função no fortalecimento da formação para $\mathrm{AB}$ no Brasil.

\section{Conclusão}

Este pequeno relato nos permite divulgar aspectos importantes desta formação que, mesmo em um momento ainda inicial, vem propiciando encontros e encorajando as lutas pela $\mathrm{AB}$ no SUS numa conjuntura de desmonte do Sistema Único de Saúde. Ele vem se constituindo em grande processo de discussão e aprendizagem não só para os mestrandos, mas também para nós docentes de IES de todo Brasil comprometidos com a garantia do direito à saúde no Brasil. Para, além disso, apresentar um pouco desta experiência nos permitiu sistematizar uma primeira avaliação das potencialidades e dos entraves deste processo tão inovador.

\section{Referências}

1. Giovanella L. Atenção Primária à Saúde seletiva ou abrangente?. Saúde Pública (Rio de Janeiro) [periódico na internet]. 2008 [citado 2018 jun. 5]; 24(supl 1): 21-3.Disponível em: http://www.scielo.br/scielo.php?script $=\mathrm{s}$ ci arttext\&pid=S0102-

$3 \overline{11 X} 2008001300005$.

2. Gomes AP, Costa JRB, Junqueira TS, Arcuri MB, Siqueira-Batista R. Atenção primária à saúde e formação médica: entre episteme e práxis. Educação Médica (Rio de Janeiro) [periódico na internet]. 2012 [citado 2018 jun. 5]; 36(4): 541-9. Disponível em:http://www.scielo.br/pdf/rbem/v36n 4/14.pdf.

3. Jesus RA, Medina MG, Prado NML. Programa Mais Médicos: análise documental dos eventos críticos $\mathrm{e}$ posicionamento dos atores sociais. Comunicação, saúde, educação (Botucatu) [periódico na internet]. 2017 [citado 2018 jun. 20]; 21(supl 1): 124156.Disponível em: http://www.scielo .br/pdf/icse/v21s1/1807-5762-icse-21s1-1241.pdf

4. Campos GWS, Pereira JN. A Atenção Primária e o Programa Mais Médicos do Sistema Único de Saúde: conquistas e limites. Saúde coletiva (Rio de Janeiro) [periódico na internet]. 2016 [citado 2018 jul. 2]; 21(9): 265563.Disponível em: http://www.scielo.br /pdf/csc/v21n9/1413-8123-csc-21-09-26 55.pdf. 
5. Brasil. Ministério da Saúde. Portaria $\mathrm{n}^{\mathrm{o}}$ 2.436, de 21 de setembro de 2017. Aprova a Política Nacional de Atenção Básica, estabelecendo a revisão de diretrizes para a organização da Atenção Básica, no âmbito do Sistema Único de Saúde (SUS). Brasília: Ministério da Saúde; 2017.

6. Morosini MVG, Fonseca AF. Revisão da Política Nacional de Atenção Básica numa hora dessas?. Saúde Pública (Rio de Janeiro) [periódico na internet]. 2017 [citado 2018 jul. 16]; 33(1): 1-3. Disponível em: http://www.epsjv.fiocruz.br/printp df $/ 6542$.

7. ABRASCO. Aplicativos de Propostas de Cursos Novos. Mestrado Profissional em Saúde da Família. 2015.

8. Pedrosa JI, Cyrino, A. Orientações para o regente - Semana 1. [tese de mestrado]. Programa de Mestrado Profissional em Saúde da Família. Disciplina Promoção da Saúde da Fundação Oswaldo Cruz (Fiocruz) 2017. Disponível em: https://campus virtual.fiocruz.br/cursos/course/view.ph $\mathrm{p}$ ? $\mathrm{id}=64 \&$ section $=0$. 